\title{
ТВЕРДОФАЗНО-СПЕКТРОФОТОМЕТРИЧЕСКОЕ ОПРЕДЕЛЕНИЕ СЕЛЕНА(IV) С ИСПОЛЬЗОВАНИЕМ ДИТИЗОНА, ИММОБИЛИЗОВАННОГО В ПОЛИМЕТАКРИЛАТНОЙ МАТРИЦЕ
}

\author{
Н.А. Гавриленко ${ }^{1}$, Н.В. Саранчина', М.А. Гавриленко \\ ${ }^{1}$ Национальный исследовательский Томский политехнический университет \\ Российская Федерация, 634050, г. Томск, пр. Ленина 30 \\ saranchina@mail.tsu.ru \\ ${ }^{2}$ Национальный исследовательский Томский государственный университет \\ Российская Федерация, 634050, г. Томск, пр. Ленина 36
}

Поступила в редакцию 1 июля 2014 г., после исправления - 1 сентября 2014 г.

\begin{abstract}
В данной статье представлена твердофазно-спектрофотометрическая методика определения селена(IV). Предложенная методика основана на реакции селена(IV) с дитизоном, иммобилизованным в полиметакрилатной матрице, в сильнокислой среде. Показано, что взаимодействие селена(IV) с дитизоном в твердой фазе сопровождается образованием комплексного соединения с максимумом поглощения 420 нм. В качестве аналитического сигнала было выбрано изменение поглощения при длине волны 610 нм, соответствующее поглощению избытка дитизона, оставшегося после проведения реакции в полиметакрилатной матрице. Исследование влияния содержания дитизона в полиметакрилатной матрице на аналитические характеристики предлагаемой методики показало, что чувствительность определения селена(IV) возрастает с увеличением его содержания. Диапазон определяемых содержаний селена(IV) составляет от 0.1 до 0.5 мг/л с пределом обнаружения 0.09 мг/л при времени контакта анализируемого раствора с матрицей равным 60 минутам. Предложенная методика была успешно применена для определения селена(IV) в фрармацевтических витаминно-минеральных препаратах. Относительное стандартное отклонение не превышает \pm 8 \%. Предложенная методика определения селена(IV) является более экологически безопасной по сравнению с экстракционно-фотометрической и отличается простотой выполнения.
\end{abstract}

Ключевые слова: селен(IV), полиметакрилатная матрица, иммобилизованный реагент, твердофазная спектрофотометрия.

Гавриленко Наталия Айратовна - к.х.н., доцент кафедры компьютерных измерительных систем и метрологии Института кибернетики ТПУ.

Область научных интересов: оптические химические сенсоры, твердофазная спектрофотометрия, иммобилизованные реагенты.

Опубликовано более 100 печатных работ.

Саранчина Надежда Васильевна - к.х.н., доцент кафедры экологии и безопасности жизнедеятельности Института неразрушающего контроля ТПУ.

Область научных интересов: иммобилизованные реагенты, комплексообразование в твердой фазе, твердофазная спектрофотометрия.

Опубликовано 38 печатных работ.

Гавриленко Михаил Алексеевич - к.Х.н., ведущий научный сотрудник лаборатории химической экологии химического факультета ТГУ.

Область научных интересов: сорбенты, хелатные комплексы, хроматография.

Опубликовано более 150 научных работ.

\section{ВВЕДЕНИЕ}

С момента открытия интерес к селену неуклонно возрастал, и расширялась область его применения. Как необходимый для живого организма, в том числе и для человека, микроэлемент селен идентифицировали относительно недавно. Особенность селена заключается в его двойственном влиянии на живые организмы. Недостаточное поступление элемента в организм приводит к дефициту селена для нормального обмена веществ, высокие концентрации проявляют токсичное действие. При этом существует очень низкая разница между необходимым и токсичным уровнем селена [1]. В настоящее время селен и его соединения широко используются в медицине и косметологии, фарма- 
цевтическая промышленность производит большое количество витаминных препаратов и биологически активных добавок с содержанием селена для преодоления селеновой недостаточности. Поэтому контроль содержания селена в различных объектах окружающей среды, пищевых продуктах и фармацевтических препаратах является чрезвычайно важной задачей и обусловлен высокой биологической активностью его соединений. Вместе с тем растет потребность в простом и селективном определении низких концентраций селена.

В работе [2] показана применимость широкого круга методов определения селена. Спектрофотометрический метод является весьма распространенным и наиболее часто используемым в аналитических лабораториях вследствие его доступности, экспрессности определения, простоты эксплуатации, легкой адаптации для массовых анализов. В работах [3-6] показана возможность спектрофотометрического определения селена в различных типах вод, мультивитаминных и мультиминеральных фрармацевтических препаратах, косметических продуктах.

В последнее время в практической аналитической химии широкое применение находит сорбционно-спектроскопический метод, позволяющий сочетать концентрирование и отделение элемента с его последующим определением непосредственно в твердой фазе. Это дает возможность повысить чувствительность определения, снизить мешающее влияние сопутствующих элементов, присутствующих в объекте анализа, снизить потребление органических растворителей, автоматизировать процесс определения [7]. Широкий выбор твердых носителей для иммобилизации реагентов и проведения реакции, наибольший интерес из которых представляют оптически прозрачные носители, обеспечивающие возможность спектрофотометрического измерения оптического сигнала, способствует развитию этих методов и их практическому использованию. Кроме того, использование твердых носителей с иммобилизованными реагентами позволяет разработать на их основе простые, дешевые, не требующие лабораторного оборудования методики для экспрессного определения веществ, в том числе и селена [8, 9].

Настоящая работа посвящена изучению возможности твердофазного спектрофотометрического определения селена (IV) с использованием дитизона, иммобилизованного в оптически прозрачной полиметакрилатной матрице.

\section{Экспериментальная часть}

Полиметакрилатную матрицу в виде прозрачной пластины толщиной $0.60 \pm 0.04$ мм получали радикальной блочной полимеризацией по методике [10]. Из исходного образца вырезали пластины размером 6.0x8.0 мм массой около 0.05 г. Рабочий 0.005 М раствор дитизона готовили в день экспе- римента растворением точной навески реагента в водном $0.005 \mathrm{M}$ растворе $\mathrm{NaOH}$. Исходные растворы ионов $\mathrm{Se}(\mathrm{IV})$ и металлов с содержанием $1 \mathrm{mr/}$ см $^{3}$ готовили в соответствии ГОСТ 4212-76 [11]. Для приготовления раствора селена(IV) использовали металлический селен, растворы меди(II) и цинка(II) готовили из препаратов $\mathrm{CuSO}_{4} \cdot 5 \mathrm{H}_{2} \mathrm{O}$ и $\mathrm{ZnSO}_{4} \cdot 7 \mathrm{H}_{2} \mathrm{O}$ квалификации «х.ч.» или «ч.д.а.». Также в работе использовали ГСО с концентрацией ионов селена(IV), меди(II) и цинка(II) 1 мг/см³. Рабочие растворы с меньшими концентрациями готовили разбавлением исходных растворов дистиллированной водой в день эксперимента. Необходимое значение $\mathrm{pH}$ создавали растворами $\mathrm{HCl}$ и контролировали стеклянным электродом на иономере И-160. Все измерения проводили при температуре $22 \pm 3^{\circ} \mathrm{C}$. Реактивы, используемые в работе, имели квалифрикацию «х.ч.» или «ч.д.а.» и применялись без дополнительной очистки.

Иммобилизацию дитизона в полиметакрилатную матрицу проводили из раствора в статическом режиме в течение 3-10 мин. После контакта с раствором дитизона матрица окрашивалась в желто-оранжевый цвет вследствие того, что дитизон в матрице находится в енольной форме [12]. Количество сорбированного в матрицу дитизона (а, мг/г) рассчитывали по формуле: $a=(c-[c]) V / m$, где $c$ и [c] - исходная и остаточная концентрации реагента в растворе, мг/л; $V$ - объем раствора, л; $m$ - масса полиметакрилатной матрицы, г. Концентрацию дитизона в растворе определяли по собственному светопоглощению при 420 нм до и после контакта с матрицей.

Взаимодействие селена(IV) с реагентом, иммобилизованным в полиметакрилатную матрицу, изучали в статических условиях. Для этого матрицу с иммобилизованным реагентом помещали в 50 мл раствора селена(IV) с различной концентрацией и значением $\mathrm{pH}$, перемешивали в течение 15-150 мин и регистрировали спектры поглощения или измеряли оптическую плотность $A$ в максимуме полосы поглощения.

Спектры поглощения и оптические плотности полиметакрилатной матрицы измеряли с помощью спектрофотометров Shimadzu UV mini - 1240 и Spekol 21. Оптические характеристики полиметакрилатной матрицы с иммобилизованным дитизоном после контакта с раствором селена(IV) измеряли относительно немодифицированной полиметакрилатной матрицы.

\section{Результаты и их обсуждение}

Дитизон является чувствительным реагентом и применим для спектрофотометрического определения широкого круга элементов, в том числе и селена. Экстракционно-фоотометрическое определение селена дитизоном происходит при экстракции селена четыреххлористым углеродом из сильнокислых сред с образованием дитизоната желто- 
го цвета с максимумом поглощения при 405-420 нм [13]. В связи с этим твердофразную экстракцию селена(IV) в полиметакрилатную матрицу с иммобилизованным дитизоном проводили из растворов хлороводородной кислоты.

Полиметакрилатная матрица с иммобилизованным дитизоном после контакта с растворами селена(IV) различной концентрации в среде $\mathrm{HCl}$ окрашивается в желто-зеленый цвет. С увеличением концентрации селена(IV) в анализируемом растворе преобладает желтый цвет матрицы. На спектрах поглощения (рис. 1) видно, что регистрируются два максимума поглощения при 420 и 610 нм. Окрашивание матрицы в желтый цвет и максимум поглощения при длине волны 420 нм соответствует образовавшемуся в матрице соединению селена с дитизоном. Зеленая окраска матрицы и максимум поглощения при длине волны 610 нм характерны для кето-фрормы не вступившего в реакцию реагента. Кроме того, максимум поглощения при 420 нм может соответствовать не только образовавшемуся в матрице дитизонату, но и енольной форме реагента, поскольку иммобилизованный дитизон при контакте с кислыми средами в полиметакрилатной матрице существует в двух таутомерных формах [12]. Из спектров поглощения видно, что наибольшая чувствительность достигается при 610 нм, поэтому в качестве аналитического сигнала была выбрана оптическая плотность при этой длине волны.

Результаты исследования влияния кислотности анализируемого раствора на изменение аналитического сигнала $\left(\Delta A_{610}\right)$ представлены на рис. 2 . Величину $\Delta A_{610}$ рассчитывали как $\Delta A_{610}=A_{0}-A$, где $A_{0}$ и $A$ - оптическая плотность полиметакрилатной матрицы с иммобилизованным реагентом после контакта с раствором в отсутствие и присутствии селена(IV) соответственно. С увеличением содержания $\mathrm{HCl}$ в анализируемом растворе изменение аналитического сигнала возрастает и становится максимальным и практически неизменным при концентрации кислоты в растворе 2.5-3.0 М.

При изучении зависимости аналитического сигнала от времени контакта полиметакрилатной матрицы с анализируемым раствором установлено, что постоянный сигнал при концентрации селена(IV) в растворе $0.5 \mathrm{mг} / л$ достигается в течение 60 мин.

Содержание дитизона в полиметакрилатной матрице влияет на ее оптическую плотность после контакта с раствором селена(IV) и определяет интервал линейности зависимости аналитического сигнала от концентрации селена(IV) в анализируемом растворе. Содержание реагента в полиметакрилатной матрице варьировали путем изменения времени контакта матрицы с раствором реагента. С увеличением содержания реагента в матрице происходит уменьшение диапазона линейной зависимости и повышение чувствительности определения селена(IV). На рис. 3 и в табл. 1 показано влияние содержания реагента в матрице на зави-

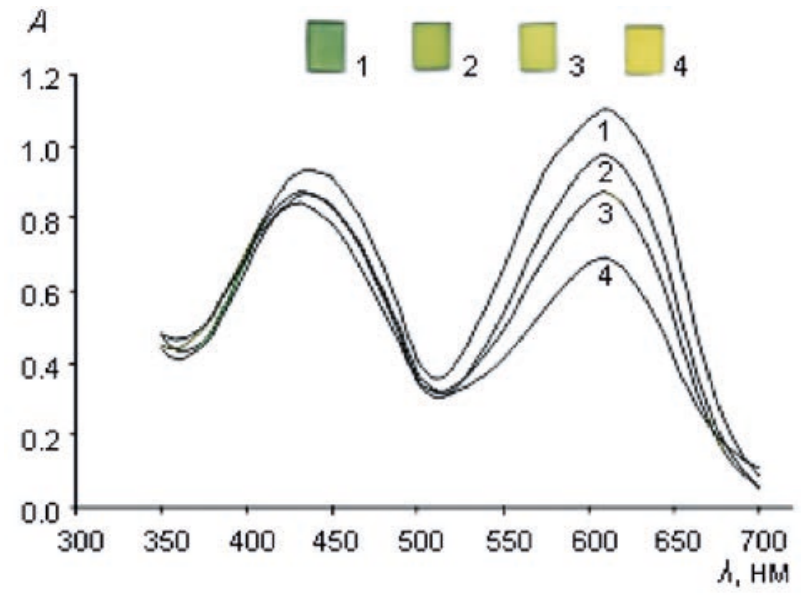

Рис. 1. Спектры поглощения и образцы полиметакрилатной матрицы с иммобилизованным дитизоном после контакта с раствором селена(IV). $C_{\mathrm{Se}(\mathrm{IV})}$ мг/л: 1 - 0; $2-0.10 ; 3-0.50 ; 4-0.75$

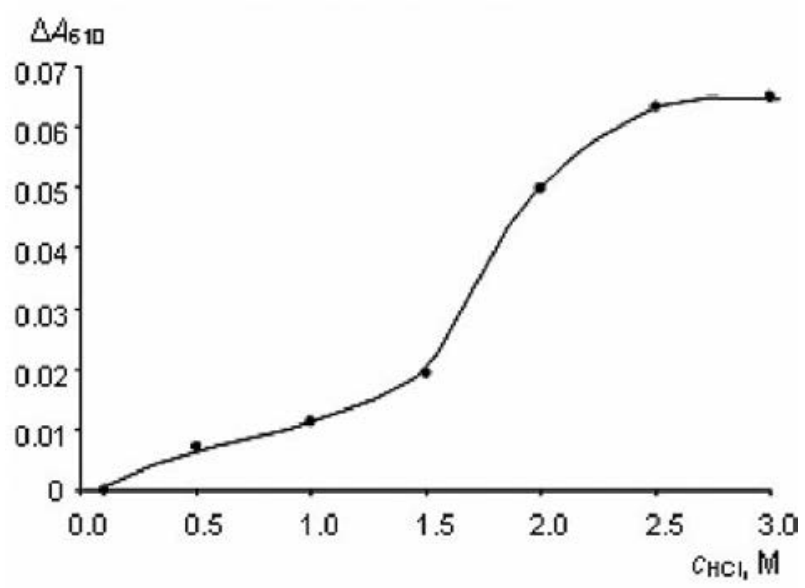

Рис. 2. Влияние содержание кислоты на изменение аналитического сигнала, $C_{\mathrm{Se}(\mathrm{IV})}=0.5 \mathrm{mr} / л$

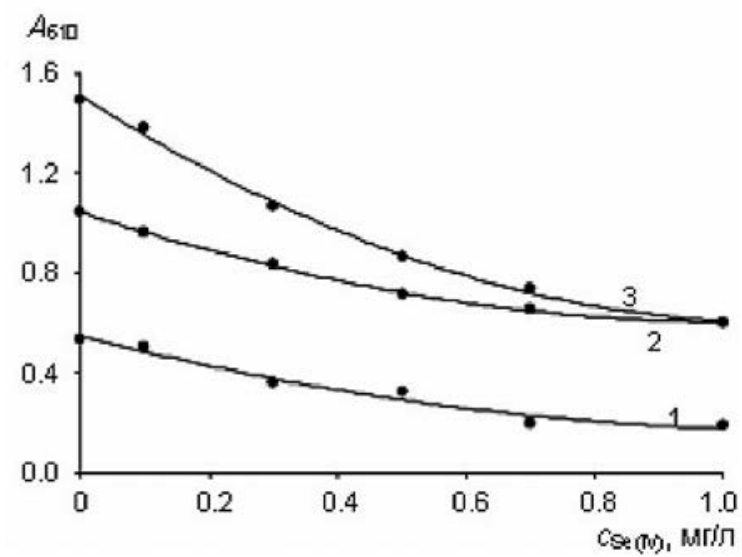

Рис. 3. Зависимость оптической плотности полиметакрилатной матрицы с иммобилизованным дитизоном от концентрации селена(IV) в растворе при содержании дитизона в матрице (мг/г): $1-0.50,2-0.65,3-0.84$ 
Таблица 1

Влияние содержания реагента в полиметакрилатной матрице на параметры градуировочной зависимости

\begin{tabular}{|c|c|c|c|c|}
\hline $\begin{array}{c}\text { Время иммобили- } \\
\text { зации реагента, } \\
\text { мин }\end{array}$ & $\begin{array}{c}\text { Содержание реаген- } \\
\text { та в матрице, мг/г }\end{array}$ & $\begin{array}{c}\text { Уравнение градуировоч- } \\
\text { ной зависимости }\end{array}$ & $\begin{array}{c}\text { Коэффрициент } \\
\text { корреляции }\end{array}$ & $\begin{array}{c}\text { Диапазон линейности } \\
\text { градуировочной зави- } \\
\text { симости, мг/л }\end{array}$ \\
\hline 3 & 0.50 & $A_{610}=0.54-0.48 c_{\mathrm{se}}$ & 0.988 & $0 \ldots 0.7$ \\
\hline 6 & 0.65 & $A_{610}=1.04-0.66 c_{\mathrm{Se}}$ & 0.997 & $0 \ldots 0.5$ \\
\hline 10 & 0.84 & $A_{610}=1.50-1.31 c_{\mathrm{Se}}$ & 0.995 & $0 \ldots 0.5$ \\
\hline
\end{tabular}

Примечания: $c_{\mathrm{Se}}$ - концентрация селена(IV) в анализируемом растворе, мг/л.

симость аналитического сигнала от концентрации селена(IV) в растворе и на параметры градуировочных зависимостей. На основании представленных результатов иммобилизацию реагента в полиметакрилатную матрицу проводили в течение 10 мин для последующего достижения наибольшей чувствительности определения селена(IV). В выбранных условиях предел обнаружения селена(IV), рассчитанный по 3s-критерию, равен 0.09 мг/л.

На основании проведенных исследований разработана твердофазно-спектрофотометрическая методика определения селена(IV) с использованием дитизона иммобилизованного в полиметакрилатной матрице.

Выполнение определения Se(IV). В раствор объемом 50 мл, содержащий аликвотную часть про-

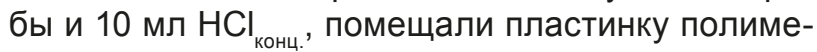
такрилатной матрицы с иммобилизованным дитизоном и перемешивали в течение 60 мин, затем вынимали, подсушивали фильтровальной бумагой и измеряли поглощение при 610 нм. Содержание селена(IV) находили по градуировочной зависимости, построенной в аналогичных условиях.

Разработанную методику опробовали на витаминных препаратах, поскольку определение селена(IV) в выбранных объектах является актуальной задачей. Предварительно исследовали мешающее влияние минеральных компонентов $\mathrm{Cu}(\mathrm{II})$ и Zn(II), содержащихся во многих витаминно-минеральных комплексах и образующих с дитизоном в кислых средах окрашенные комплексные соединения [14]. Погрешность определения селена(IV) в присутствии посторонних ионов рассчитывали по формуле: $\delta(\%)=\left[\left(A_{i}-A\right) / A\right] \cdot 100$, где $A$ и $A_{i}-$ оптическая плотность полиметакрилатной матрицы с иммобилизованным реагентом после контакта с раствором селена(IV) в отсутствие и в присутствии постороннего иона соответственно. Показано, что при введении в анализируемый раствор 10-кратных количеств ионов Zn(II) и Cu(II) погрешность определения селена составляет -17\% и 42 \% соответственно. При введении равных количеств погрешность в присутствии меди остается значительной и составляет $28 \%$, в присутствии цинка уменьшается до -5 \%. Мешающее действие Zn(II) можно устранить введением в анализируемый раствор двунатриевой соли этилендиаминтетрауксусной кислоты, Сu(II) - введением 1,10-френантроли- на в соотношениях металл : маскирующий агент = $1: 150$ и $1: 200$ соответственно. Проведенные эксперименты показали, что в присутствии маскирующих агентов погрешность определения селена в присутствии 10-кратных количеств Zn(II) и $\mathrm{Cu}(\mathrm{II})$ снижается до $\pm 4 \%$.

В выбранных условиях определения селена(IV) вступать в реакцию с дитизоном и образовывать окрашенные комплексные соединения могут также $\mathrm{Au}(\mathrm{III}), \mathrm{Pd}(\mathrm{II}), \mathrm{Pt}(\mathrm{II}), \mathrm{Hg}(\mathrm{II}), \mathrm{Ag}(\mathrm{I}), \mathrm{Te}(\mathrm{IV})$ [14]. При контроле $\mathrm{Se}(\mathrm{IV})$ в объектах, содержащих растворимые формы этих элементов, необходимо устранять их мешающее влияние предварительным отделением или введением в анализируемый раствор маскирующих агентов.

Методика опробована при определении селена(IV) в витаминно-минеральном комплексе с антиоксидантами «Селмевит» и витаминно-минеральном комплексе «Компливит селен». Указанные препараты в своем составе содержат селен в виде натрия селенита, анализируемые растворы препаратов готовили растворением шести таблеток «Селмевита» и трех таблеток «Компливит селена» в мерных колбах вместимостью 25 мл. Для анализа отбирали по 10 мл полученных растворов и поступали согласно описанной последовательности выполнения определения. Выбранные препараты в своем составе содержат Cu(II) и Zn(II), поэтому в анализируемые растворы добавляли маскирующие агенты.

Результаты определения селена(IV) в витаминных препаратах представлены в табл. 2. Правильность предлагаемой методики оценивали по результатам определения добавки селена(IV) в пробах витаминных препаратов. Для оценки правильности использовали показатель правильности (б), представляющий собой выраженное в процентах отношение разности найденной концентрации (среднего значения) и введенного значения концентрации к введенному значению. Прецизионность результатов анализа в условиях повторяемости оценивали по величине относительного стандартного отклонения $\left(s_{r}\right)$. Полученные результаты определения $\mathrm{Se}(\mathrm{IV})$ правильные, и прецизионность результатов анализа в условиях повторяемости сопоставима с данной характеристикой спектрофотометрических методик анализа растворов, величина $s_{r}$ не превышает значений 0.06-0.08. 
Результаты определения селена(IV) и добавок селена(IV) в витаминных препаратах $(n=3-5 ; P=0.95)$

\begin{tabular}{|c|c|c|c|c|c|c|c|}
\hline \multirow{2}{*}{ Препарат } & \multirow{2}{*}{$\begin{array}{c}\text { Указано на } \\
\text { упаковке, } \\
\text { мкг/табл. }\end{array}$} & \multirow{2}{*}{$\begin{array}{l}\text { Найдено, } \\
\text { мкг/табл. }\end{array}$} & \multirow[b]{2}{*}{$s_{r}$} & \multicolumn{2}{|c|}{ Добавка } & \multirow[b]{2}{*}{$s_{r}$} & \multirow{2}{*}{$\sigma, \%$} \\
\hline & & & & $\begin{array}{l}\text { Введено, } \\
\text { мкг/табл. }\end{array}$ & $\begin{array}{l}\text { Найдено, } \\
\text { мкг/табл. }\end{array}$ & & \\
\hline «Селмевит» & 25 & $26.1 \pm 1.2$ & 0.04 & 20 & $20.9 \pm 1.6$ & 0.06 & 4.4 \\
\hline «Компливит селен» & 70 & $67 \pm 4$ & 0.03 & 50 & $53 \pm 3$ & 0.08 & 5.8 \\
\hline
\end{tabular}

Примечание: табл. - таблетка.

\section{Заключение}

Показана возможность использования полиметакрилатной матрицы с иммобилизованным дитизоном для твердофазно-спектрофотометрического определения 0.1-0.5 мг/л селена(IV) с пределом обнаружения 0.09 мг/л при объеме анализируемой пробы 50 мл. Предложенная методика отличается простотой выполнения, показана ее применимость к анализу витаминно-минеральных комплексов на содержание селена(IV).

Работа выполнена при фринансовой поддержке Российского научного фронда в рамках гранта № 14-19-00926.

\section{ЛИТЕРАТУРА}

1. Ермаков В.В., Ковальский В.В. Биологическое значение селена. М.: Наука, 1974. 298 с.

2. Аналитический контроль содержания селена в природных водах / А.Р. Гарифзянов [и др.] // Заводская лаборатория. Диагностика материалов. 2001. Т. 67, № 1. С. 3-15. 3. Agrawal K., Patel K.S., Shirivas K. Development of surfactant assisted spectrophotometric method for determination of selenium in waste water samples // Journal of Hazardous Materials. 2009. V. 161. P. 1245-1249.

4. Tantaru G., Vieriu M., Popescu M.-C. Validation of spectrophotometric method for Se(IV) determination: analytical applications. Environ Monit Assess. 2014. V. 186. P. 3277-3282. 5. Sankalia J.M., Mashru R.C., Sankalia M.G. Spectroscopic determination of traice amounts of selenium(IV) in multivitamin with multimineral formulations using 3,3'-diaminobenzidine hydrochloride // Spectroscopy Letters. 2005. V. 38. № 1. P. 61-76.

6. Kumar K.S., Suvardhan K., Kang S.H. Facile and Sensitive Determination of Selenium (IV) in Pharmaceutical Formulations by Flow Injection Spectrophotometry // Journal of Pharmaceutical Sciences. 2008. V. 97. № 5. P. 1927-1931.

7. Solid phase extraction for the speciation and preconcentration of inorganic selenium in water samples / C.H. Latorre [et al.] // Analytica Chimica Acta. 2013. V. 804. P. 37-49.

8. З Запорожец О.А., Билоконь С.Л., Запорожец О.А. Визуальный тест-метод определения селена(IV), иммобилизованным на кремнеземе индигокармином // Журн. аналит. химии. 2007. Т. 62, № 2. С. 208-212.

9. Амелин В.Г., Королева О.В. Модифицированные органическими реагентами ткани и бумаги для тестопределения селена(IV) и теллура(IV) // Журн. аналит. химии. 2009. Т. 64, № 12. С. 1304-1308.

10. Индикаторный чувствительный материал для определения микроколичеств веществ / Гавриленко Н.А., Мокроусов Г.М. Пат. 2272284 Рос. Федерация; № 2004125304/04; заявл. 18.08.2004; опубл. 20.03.2006. Бюл. № 8.9 с.

11. ГОСТ 4212-76. Реактивы. Методы приготовления растворов для колориметрического и нефелометрического анализа. М.: Стандартинформ, 2008. 22 с.

12. Саранчина Н.В. Аналитические свойства дитизона и диэтилдитиокарбамината, иммобилизованных в полиметакрилатную матрицу. Дис. ... канд. хим. наук. Томск, 2007. 138 с.

13. Назаренко И.И., Ермаков А.Н. Аналитическая химия селена и теллура. М.: Наука, 1971. 251 с.

14. Sandell E.B. Colorimetric Determination of Traces of Metals. New York: Interscience, 1959. 682 p.

\title{
SOLID PHASE SPECTROPHOTOMETRIC DETERMINATION OF SELENIUM(IV) USING DITHISONE IMMOBILIZED IN A POLYMETHACRYLATE MATRIX
}

\author{
N.A. Gavrilenko ${ }^{1}$, N.V. Saranchina ${ }^{1}$, M.A. Gavrilenko ${ }^{2}$ \\ ${ }^{1}$ Federal State Autonomous Educational Institution of \\ Higher Education «National Research Tomsk Polytechnic University» \\ pr. Lenina, 30, Tomsk, 634050, Russian Federation \\ saranchina@mail.tsu.ru \\ ${ }^{2}$ Federal State Autonomous Educational Institution of \\ Higher Education «National Research Tomsk State University» \\ pr. Lenina, 36, 634050, Tomsk, Russian Federation
}

This paper presents a solid-phase spectrophotometric method for the selenium(IV) determination. The proposed method is based on the reaction between selenium(IV) and immobilized into transparent polymethacrylate matrix dithizone in strongly acidic solution. It was shown that the interaction of 
selenium(IV) with dithizone in solid phase was accompanied by the formation of the complex with a $420 \mathrm{~nm}$ absorption maximum. As an analytical signal, the change of absorption at $610 \mathrm{~nm}$ wavelength corresponding to the absorption of excess dithizone remaining after the reaction in polymethacrylate matrix was chosen. The investigation of an effect of dithizone content in polymethacrylate matrix on the analytical characteristics of the proposed method showed that the sensitivity of the selenium(IV) determination increases with its contents. Range of values for the determined concentrations are $0.1 \ldots 0.5$ $\mathrm{mg} / \mathrm{L}$ with the limit of detection $0.09 \mathrm{mg} / \mathrm{L}$ where the time of the contact of the sample solution with the polymethacrylate matrix is $60 \mathrm{~min}$. The proposed method was successfully applied to the determination of selenium(IV) in pharmaceutical multivitamin and mineral formulations. Statistical analysis of the results confirms the precision and accuracy of the proposed method. The relative error of determination does not exceed $\pm 8 \%$. The developed method is ecological, cost-effective and involves easily accessible instrumentation technique which can be used by ordinary research laboratories.

Keywords: selenium(IV), polymethacrylate matrix, immobilized reagent, solid-phase spectrophotometry.

\section{REFERENCES}

1. Ermakov V.V., Koval'skii V.V. Biologicheskoe znachenie selena [The biological importance of selenium]. Moscow, Nauka Publ., 1974. 298 p. (in Russian)

2. Garifzianov A.R., Budnikov G.K., Toropova V.F., Gainutdinova D.F. [Analytical control of selenium in natural waters]. Zavodskaia laboratoriia. Diagnostika materialov [Industrial laboratory. Diagnostics of materials], 2001, vol. 67, no. 1, pp. 3-15 (in Russian)

3. Agrawal K., Patel K.S., Shirivas K. Development of surfactant assisted spectrophotometric method for determination of selenium in waste water samples // Journal of Hazardous Materials, 2009, vol. 161, pp. 1245-1249. doi: 10.1016/j. jhazmat.2008.04.082

4. Tantaru G., Vieriu M., Popescu M.-C. Validation of spectrophotometric method for Se(IV) determination: analytical applications. Environ Monit Assess, 2014, vol. 186, pp. 32773282. doi: 10.1007/s10661-014-3618-8

5. Sankalia J.M., Mashru R.C., Sankalia M.G. Spectroscopic determination of traice amounts of selenium(IV) in multivitamin with multimineral formulations using 3,3'-diaminobenzidine hydrochloride // Spectroscopy Letters, 2005, vol. 38, no 1, pp. 61-76. doi: 10.1081/SL-200045466

6. Kumar K.S., Suvardhan K., Kang S.H. Facile and Sensitive Determination of Selenium (IV) in Pharmaceutical Formulations by Flow Injection Spectrophotometry // Journal of Pharmaceutical Sciences, 2008, vol. 97, no 5, pp. 1927-1931.

7. Latorre C.H., García J.B., Martín S.G., Pena Crecente R.M. Solid phase extraction for the speciation and preconcentration of inorganic selenium in water samples // Analyt- ica Chimica Acta, 2013, vol. 804, pp. 37-49. doi: 10.1016/j. aca.2013.09.054

8. Zaporozhets O.A., Bilokon' S.L., Zaporozhets O.A. [The visual test method for determination of selenium (IV), immobilized on silica indigokarmina]. Zhurnal analiticheskoi khimii [Journal of Analytical Chemistry], 2007, vol. 62, no. 2, pp. 208-212 (in Russian)

9. Amelin V.G., Koroleva O.V. [Fabrics and papers modified with analytical reagents for the test determination of selenium(IV) and tellurium(IV)]. Zhurnal analiticheskoi khimii [Journal of Analytical Chemistry], 2009, vol. 64, no. 12, pp. 1304-1308 (in Russian)

10. Gavrilenko N.A., e.a. Indikatornyi chuvstvitel'nyi material dlia opredeleniia mikrokolichestv veshchestv [Indicating sensitive material for determination of trace substances]. Patent RF, no. 2272284, 2006. (in Russian)

11. GOST 4212-76. Reaktivy. Metody prigotovleniia rastvorov dlia kolorimetricheskogo i nefelometricheskogo analiza [State Standard 4212-76. Reagents. Methods for the preparation of solutions for the colorimetric and nephelometric analysis]. Moscow, Standartinform Publ., 2008. 22 p. (in Russian) 12. Saranchina N.V. Analiticheskie svoistva ditizona i dietilditiokarbaminata, immobilizovannykh $v$ polimetakrilatnuiu matritsu. Dis. ... kand. khim. nauk [Analytic properties of dithizone and dietilditiokarbaminata immobilized in a polymethacrylate matrix. Cand. chem. Sci. diss.]. Tomsk, 2007. 138 p. (in Russian)

13. Nazarenko I.I., Ermakov A.N. Analiticheskaia khimiia selena i tellura [Analytical chemistry of selenium and tellurium]. Moscow, Nauka Publ., 1971. 251 p. (in Russian)

14. Sandell E.B. Colorimetric Determination of Traces of Metals. New York, Interscience Publ, 1959. 682 p. 